\title{
Properties of ideal Gaussian glass-forming systems
}

\author{
Andreas Heuer and Aimorn Saksaengwijit* \\ University of Münster, Institute of Physical Chemistry, \\ Corrensstr. 30, D-48149 Münster, Germany \\ *King Mongkut's University of Technology Thonburi, Thailand
}

(Dated: August 18, 2021)

\begin{abstract}
We introduce the ideal Gaussian glass-forming system as a model to describe the thermodynamics and dynamics of supercooled liquids on a local scale in terms of the properties of the potential energy landscape (PEL). The first ingredient is the Gaussian distribution of inherent structures, the second a specific relation between energy and mobility. This model is compatible with general considerations as well as with several computer simulations on atomic computer glass-formers. Important observables such as diffusion constants, structural relaxation times and kinetic as well as thermodynamic fragilities can be calculated analytically. In this way it becomes possible to identify a relevant PEL parameter determining the kinetic fragility. Several experimental observations can be reproduced. The remaining discrepancies to the experiment can be qualitatively traced back to the difference between small and large systems.
\end{abstract}

PACS numbers: 64.70.Pf 


\section{INTRODUCTION}

The understanding of the dynamics of supercooled liquids is still far from being complete [1, 2, 3, 4]. A lot of insight has been gained from simulations . For example, in real space the microscopic nature of dynamic heterogeneities has been clarified [ [5, 6, 7, 8, ㅁ, 10, 11]. Using the framework of the potential energy landscape (PEL) a lot of insight could be also

gained in configuration space [12, 13]. A key aspect is the use of inherent structures (IS), i.e. local minima of the PEL. Upon minimization basically all configurations can be mapped on a IS. In this way the regular dynamics can be mapped on a hopping dynamics between IS [14, 15]. Physically, this mapping can be interpreted as a removal of the vibrational degrees of freedom. However, as explicitly shown in [16] the properties of the structural relaxation remain identical for sufficiently low temperatures. Generally speaking, the mapping on the IS can be interpreted as a coarse-graining procedure. At low temperatures the IS dynamics displays many correlated forward-backward jumps between adjacent IS. In a further coarsegraining step it is possible to define metabasins (MB) by an appropriate merging of adjacent IS [17, 18, 19, 20, 21]. In this way the effect of correlated forward-backward motion has basically disappeared.

A key question deals with the relation between thermodynamics and dynamics. For example the empirical Adam-Gibbs relation [22]

$$
\Gamma(T)=\Gamma_{0} \exp \left(-B_{A G} / T s_{c}(T)\right)
$$

relates the configurational entropy $s_{c}$ to the local relaxation rate $\Gamma$. A further relation between thermodynamics and dynamics is formulated via the fragilities. In the spirit of the thermodynamic fragility as discussed in [23, 24] one can define the thermodynamic fragility index via [25]

$$
m_{\text {thermo }}=-\beta_{g} \frac{S_{c}^{\prime}\left(\beta_{g}\right)}{S_{c}\left(\beta_{g}\right)} .
$$

where $T_{g}=1 / \beta_{g}$ (choosing $\left.k_{B}=1\right)$ denotes the glass-transition temperature. Furthermore, the kinetic fragility is defined as

$$
m_{k i n}=d \ln \tau_{\alpha} / d\left(T_{g} / T\right)
$$

Qualitatively, it denotes the slope of the relaxation time (or viscosity) in the Angell-plot [23, 26]. Empirically, one finds a significant correlation between the kinetic and the thermodynamic fragility [23]. In principle the kinetic fragility may also be defined for the 
diffusion constant. Due to the violation of the Stokes-Einstein relation [27] minor variations of the value of $m_{k i n}$ will be present. Furthermore, it turns out that for the set of all glass-forming systems one observes a significant correlation between $m_{k i n}$ and the degree of non-exponentiality, expressed, e.g., by the exponent $\beta_{K W W}$ of the stretched exponential function [28]. If one restricts oneself, however, to the set of all molecular glass-forming systems (excluding in particular network forming systems and polymers) the residual correlation is very weak $(-0.28)$ and the values of $\beta_{K W W}$ are restricted for most of the systems $(>80 \%)$ in that work to a relatively small regime between 0.5 and 0.62 [28, 29]. In contrast, the network-forming systems are characterized by nearly exponential relaxation and small values of $m_{k i n}$.

In the language of the IS or the MB the thermodynamic properties at constant volume are to large extent determined by their energy distribution $G(e)$. For many systems it has been shown numerically that the distribution of IS can be described by a Gaussian [30, 31, 32, 33]. Even for BKS-SiO ${ }_{2}$ the distribution is Gaussian, albeit displaying a low-energy cutoff in the range of accessible temperatures for computer simulations [34]. Furthermore, it turns out that the distribution of IS and MB is nearly identical in the relevant regime of low-energy states [20].

Within the PEL approach it is possible to relate the thermodynamic and the dynamic aspects [19, 20, 21, 35]. This is based on the observation that the escape rate from a MB can be expressed in terms of its energy, i.e. $\Gamma(e, T)$. Furthermore, it turns out that the temperature dependence of the diffusion constant $D(T)$ can be fully expressed in terms of the average local escape rate. As a consequence, knowledge of $G(e)$ and $\Gamma(e, T)$ allows one to predict $D(T)$. A similar type of relation between energy and mobility can be found, e.g., for the trap model [36].

The goal of this work is to elucidate the properties of a system with a Gaussian distribution $G(e)$ of $\mathrm{MB}$. The functional form of $\Gamma(e, T)$ is rationalized by different models, discussed in literature, and at the same time by comparison with previous computer simulations on the binary mixture Lennard-Jones system (BMLJ) and silica $\left(\mathrm{BKS}-\mathrm{SiO}_{2}\right)$. On this basis we define an ideal Gaussian glass-former (IGGF). For the IGGF several observables can be determined analytically such as the temperature dependent diffusion constant and relaxation time, its kinetic and thermodynamic fragility and its non-exponentiality. In this way it becomes possible, e.g., to elucidate the relevant PEL parameters which determine the 
fragility. In Sect.II the IGGS is introduced and in Sect.III its main properties are calculated. We end with a critical discussion and a summary in Sect.IV.

\section{DESCRIPTION OF THE IDEAL GAUSSIAN GLASS-FORMER}

\section{A. Thermodynamics}

Of crucial importance for the properties of a glass-forming system is the number density of IS, denoted $G(e)$. Here we always consider a system with $N$ particles. For many different systems, studied via computer simulations, a Gaussian density of IS has been found [32, 33, 37, 38], i.e.

$$
G(e)=\exp (\alpha N) \frac{1}{\sqrt{2 \pi \sigma^{2}}} \exp \left(-\left(e-e_{0}\right)^{2} / 2 \sigma^{2}\right) .
$$

A notable exception is $\mathrm{BKS}-\mathrm{SiO}_{2}$. This system is characterized by a low-energy cutoff [39] which gives rise to the fragile-to-strong crossover [39, 40]. In principle, for the calculations, shown below, the effect of a low-energy cutoff can be incorporated [29]. Here we mainly concentrate just on the case of a purely Gaussian density of IS.

For a closer discussion one has to take into account that the average curvature around the minima may depend on $e$. For different systems it turns out to a very good approximation that one has a linear energy-dependence for the free energy $F_{\text {harm }}(e)$, related to the harmonic vibration in a well [30, 31, 32, 41, 42, 43, 44]. This can be written as

$$
F_{\text {harm }}(e)=\text { const }-\beta_{\text {harm }} e .
$$

The constant $\beta_{\text {harm }}$ is a material constant. The meaning of the sign of $\beta_{\text {harm }}$ is visualized in Fig.1.

The Boltzmann distribution $p_{e q}(e)$ describes the probability to be (at a randomly chosen time) in an IS with energy e. $p_{e q}(e)$ is proportional to $G(e) \exp (-\beta e)$ when $\beta_{\text {harm }}=0$. Taking into account the curvature-effect, introduced above, one finds

$$
p_{e q}(e) \propto G_{e f f}(e) \exp (-\beta e)
$$

with the effective density

$$
G_{e f f}(e) \propto G(e) \exp \left(-F_{\text {harm }}(e)\right) \propto \exp \left(-\left(e-e_{0, e f f}\right)^{2} / 2 \sigma^{2}\right) .
$$




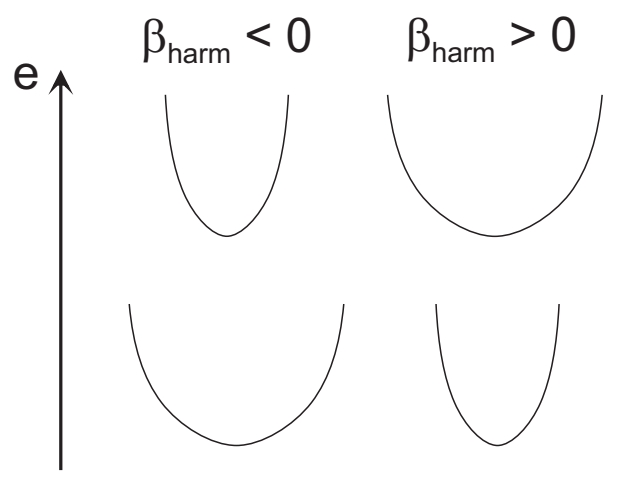

Figure 1: A sketch of the cases $\beta_{\text {harm }}<0$ and $\beta_{\text {harm }}>0$. Shown are typical curvatures around representative IS at different energies.

and $e_{0, e f f}=e_{0}+\beta_{\text {harm }} \sigma^{2}$. Thus the presence of an energy-dependent average curvature can be incorporated by a shift of the Gaussian distribution of states.

The standard definition of the configurational entropy is $-\sum_{i} p_{i} \ln p_{i}$ where the sum is over all states (not energies). Mapping this relation to the description in terms of energies one obtains

$$
S_{c}(T)=\int d e p_{e q}(e) \ln \left(G(e) / p_{e q}(e)\right)=\int \operatorname{dep}_{e q}(e) S_{c}(e)-\int d e p_{e q}(e) \ln p_{e q}(e) .
$$

For the $G(e)$ and $p_{e q}(e)$, obtained for the Gaussian distribution, one obtains from the first term

$$
S_{c}(T)=N \alpha-(1 / 2) \sigma^{2}\left(\beta-\beta_{\text {harm }}\right)^{2} .
$$

For large $N$ one expects that $\sigma^{2} \propto N$ due to the central limit theorem. Then $S_{c}(T)$ becomes extensive as expected. In contrast, the last term in Eq.8, which would give rise to 1/2, can be neglected because it is not extensive and just gives rise to a minor redefinition of $\alpha$ $(\alpha \rightarrow \alpha+1 / 2 N)$.

Defining the Kauzmann-temperature by the condition $S_{c}\left(T_{K}\right)=0$ (and $\left.\beta_{K}=1 / T_{K}\right)$ Eq.9 can be equivalently expressed as

$$
T S_{c}(T)=\left[(N \alpha)+\sigma^{2} \beta \beta_{K} / 2-\sigma^{2} \beta_{\text {harm }}^{2} / 2\right]\left(T-T_{K}\right) .
$$

Neglecting the temperature-dependence of the second term this is actually the standard expression when deriving the VFT-temperature dependence (e.g. $\ln \left(D(T) / D_{0}\right) \propto 1 /\left(T-T_{0}\right)$ where often $T_{0} \approx T_{K}$ is found) from the Adam-Gibbs expression [41]. Using a similar way 


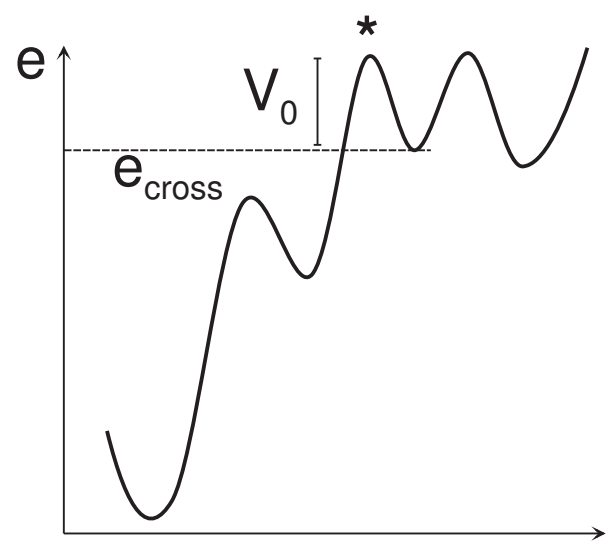

Figure 2: Sketch of the multi-step escape process, including the definition of $V_{0}$. The barrier with the star is supposed to be the critical barrier beyond which $p_{\text {back }}<0.5$.

of rewriting the configurational entropy, this type of argument can be already found in [41]. In any event, for the further analysis we will use the expression Eq9 due to its simplicity.

\section{B. Transitions between MB: Models}

There is a long history of models which describe the dynamics in configuration space on a phenomenological level [36, 45, 46, 47, 48, 49]. One considers a region of the viscous fluid which can cooperatively rearrange via a transition state. For the time being the initial and final states may be characterized by the energy of the respective IS (or MB). For sufficiently low temperatures the elementary rearrangement process is considered to be activated: the system leaves a state with energy $e$, crosses a high-energy transition state with rate $\Gamma(e)$ (from now on the variable $T$ is omitted) and ends up in a new state which is uncorrelated to the initial one. Different names can be found for essentially identical models (e.g. trap model, free energy model) following this scenario.

The hopping rate $\Gamma(e)$ is characterized by two energies. $e_{\text {cross }}$ denotes the energy of the IS just after the final barrier, which has a height $V_{0}$; see Fig 2 for the sketch. According to the model assumptions $e_{\text {cross }}$ and $V_{0}$ are independent of the initial energy $e$. Actually, even in more complex systems like the random energy model one can argue via percolation arguments that $e_{\text {cross }}$ is independent of $e$ [50]. More generally, in a percolation-like picture of the PEL $e_{\text {cross }}$ corresponds to the energy level from which on the system finds adjacent states with similar energies and thus does not have to increase further in the PEL for the 
final relaxation. Defining $E_{\text {app }}(e)$ as the apparent activation energy to escape from energy $e$, this scenario can be written as

$$
\Gamma(e)=\Gamma_{0}(e) \exp \left(-\beta E_{a p p}(e)\right)
$$

with

$$
E_{\text {app }}(e)=e_{\text {cross }}+V_{0}-e
$$

for $e \leq e_{\text {cross }}$ and $E_{\text {app }}(e)=V_{0}$ for $e \geq e_{\text {cross }}$. Stated differently, the escape for energies lower than $e_{\text {cross }}$ is solid-like (activated) whereas otherwise it is liquid-like [35].

The energy-dependent prefactor $\Gamma_{0}(e)$ reflects possible entropic effects. As argued in [45, 51] the prefactor $\Gamma_{0}(e)$ contains an energy-dependent factor $M_{\text {entro }}$ which denotes the number of escape paths to reach a high-energy state with energy $e_{\text {cross }}$. In most models this is neglected by simply choosing $\Gamma_{0}(e)=\Gamma_{0}$. This would be justified in case of $1 \mathrm{D}$ reaction paths or low-dimensional percolation paths. A simple expression for $M_{\text {entro }}$ can be formulated if every state with energy $e_{\text {cross }}$ can be reached from exactly one state with energy $e\left(<e_{\text {cross }}\right)$. It is given by $M_{\text {entro }}=G\left(e_{\text {cross }}\right) / G(e)$ [52], i.e.

$$
\Gamma_{0}(e)=\Gamma_{0} G\left(e_{\text {cross }}\right) / G(e) .
$$

This holds for $e<e_{\text {cross }}$ in the opposite limit one just has $\Gamma_{0}(e)=\Gamma_{0}$. For $e_{\text {cross }}-e \ll$ $e_{0}-e_{\text {cross }}$ Eq13 can be approximated as

$$
\Gamma_{0}(e) \approx \Gamma_{0} \exp \left(\left(e_{0}-e_{\text {cross }}\right)\left(e_{\text {cross }}-e\right) / \sigma^{2}\right)
$$

For later purposes this is rewritten as

$$
\Gamma_{0}(e)=\Gamma_{0} \exp \left(\kappa k_{\text {entro }}\left(e_{\text {cross }}-e\right)\right)
$$

with

$$
k_{\text {entro }}=\left(e_{0, \text { eff }}-e_{\text {cross }}\right) / \sigma^{2}
$$

and

$$
\kappa=\frac{e_{0}-e_{\text {cross }}}{e_{0, \text { eff }}-e_{\text {cross }}}
$$

This somewhat complicated way to rewrite Eq.14 is motivated in two ways. First, because $p_{e q}(e)$ is directly related to $G_{e f f}(e)$ and thus to $e_{0, e f f}$, see Eq 6, it is more convenient to use $e_{0, \text { eff }}$ rather than $e_{0}$. Second, in practice the factor $\kappa$ has to be treated as an empirical 


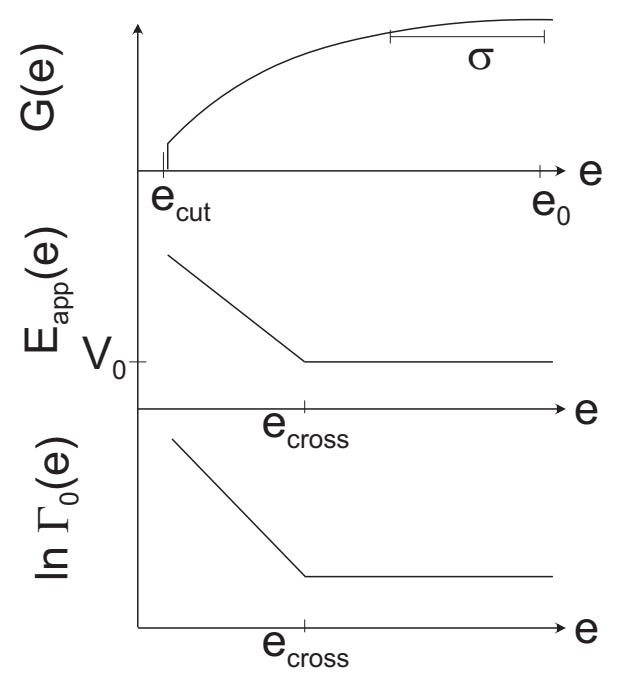

Figure 3: Sketch of the energies, introduced in the text. A possible difference between $G(e)$ and $G_{\text {eff }}(e)$ is neglected.

parameter because the increase of the entropic term $\Gamma_{0}(e)$ with decreasing energy may somewhat deviate from the specific scenario, described above. The relevant energy scales are summarized in Fig. 3 .

It is convenient to introduce the shifted inverse temperature

$$
\tilde{\beta}=\beta-\kappa k_{\text {entro }} \text {. }
$$

In principle all calculations, shown in this work, can be performed as well for $\kappa \neq 1$. However, since the influence of the entropic prefactor is not as important as the energetic term the additional complexity of the expressions is not worth the additional information for $\kappa \neq 1$. In what follows we therefore always choose $\kappa=1$.

Now one can rewrite Eq.11 as

$$
\Gamma(e)=\Gamma_{0} \exp \left(-\tilde{\beta}\left(e_{\text {cross }}-e\right)\right) \exp \left(-\beta V_{0}\right)
$$

and, for a Gaussian density of states,

$$
p_{e q}(e) \propto G_{\text {eff }}(e) \exp (-\beta e) \propto \exp \left[-\left(e-e_{\text {cross }}+\sigma^{2} \tilde{\beta}\right)^{2} / 2 \sigma^{2}\right]
$$

When comparing Eq. 19 with simulations one has to take into account that the simulated system may contain more than one elementary system. Each subsystem is characterized by an energy $e_{i}$ and $e=\sum e_{i}$. For a superposition of $M$ independent subsystems the total 
hopping rate $\Gamma_{M}(e)$ is just the sum of the individual hopping rates $\Gamma\left(e_{i}\right)$. To a first approximation one may assume that the energy $e$ is equally distributed among the $M$ subsystems, yielding $\Gamma_{M}(e)=M \Gamma(e / M)$. A closer analysis shows that apart from another energyindependent factor this is indeed the correct expression [29]. This expression for $\Gamma_{M}(e)$ suggests to generalize Eq.19 to

$$
\Gamma(e)=\Gamma_{0} \exp \left(-\lambda \tilde{\beta}\left(e_{\text {cross }}-e\right)\right) \exp \left(-\beta V_{0}\right)
$$

Here $1 / \lambda$ is a measure for the number of elementary subsystems, present in the specific system. This completes the definition of the IGGF. For later purposes we introduce the dimensionless quantity

$$
\mu \equiv \tilde{\beta} \sigma
$$

which will turn out to be the central quantity characterizing the properties of the IGGF.

\section{Comparison with simulations}

The above scenario has been compared with simulations of relatively small systems of the BMLJ system $(N=65)[20]$ and $\left.\mathrm{BKS}^{-\mathrm{SiO}_{2}}(N=99)[52]\right)$. This comparison has been performed for $\mathrm{MB}$ in order to have a random-walk type dynamics in configuration space. With IS it would have been impossible to express observables such as the diffusion constant or the relaxation time just in terms of the waiting times [20, 29]. For the comparison the average waiting time in $\mathrm{MB}$ of a given energy $e$ have been determined, denoted as $\langle\tau(e)\rangle$. Naturally, the average escape rate $\Gamma(e)$ is then given by

$$
\Gamma(e)=\frac{1}{\langle\tau(e)\rangle} .
$$

Note that this definition does not imply that the escape from a MB with energy $e$ corresponds to an exponential waiting time distribution with average waiting time $\langle\tau(e)\rangle$.

The simulations have fully confirmed the validity of Eq21 except for a slight smearing out effect for energies close to $e_{\text {cross }}$. Actually, the effective barriers could be identified by a closer analysis of the relevant minima and saddles of the PEL [20]. Actually, in [53] it has been shown that the additional barrier before the final transition (denoted $V_{0}$ above) and the barrier, governing the local forward-backward motion at low temperatures (within a MB) are roughly the same. 


\begin{tabular}{|c|c||c|c|c|c||c|c|c|c|c|}
\hline & \multicolumn{1}{|c||}{} & \multicolumn{3}{c||}{ thermodynamic } & \multicolumn{5}{c|}{ dynamic } \\
\hline & $\mathrm{N}$ & $\sigma$ & $-e_{\text {cut }}$ & $\beta_{\text {harm }}$ & $\alpha$ & $-e_{\text {cross }}$ & $\lambda$ & $\kappa$ & $V_{0}$ & $\Gamma_{0}$ \\
\hline BKS-SiO $_{2}$ & 99 & $3.5 \mathrm{eV}$ & $43.4 \mathrm{eV}$ & $\approx 0$ & 1.14 & $37.5 \mathrm{eV}$ & 0.66 & 0.62 & $0.8 \mathrm{eV}$ & $1 /(20 \mathrm{fs})$ \\
\hline BMLJ & 65 & 3.0 & - & -0.3 & 0.73 & 12.9 & 0.55 & 0.3 & 1.0 & $1 / 150$ \\
\hline
\end{tabular}

Table I: The thermodynamic and dynamic PEL parameters, obtained from simulation of $\mathrm{BKS} \mathrm{SiO}_{2}$ and BMLJ.

Very recently, de Souza and Wales have analyzed the temperature dependence of the mean square displacement, evaluated for a fixed time $\tau$ [53]. Of course, for very large $\tau$ this analysis recovers the standard diffusion coefficient. For ambient $\tau$, which for the lowest temperatures is significantly shorter than $\tau_{\alpha}$, the authors observe a simple Arrhenius behavior with the high-temperature activation energy $V_{0}$. For lower temperatures this approach is sensitive to the local forward-backward motion within a MB. The barriers in this regime are of the order of $V_{0}$ so that the local processes remain activated with the high-temperature activation energy. This strengthens the observation that it is roughly the same value $V_{0}$ which governs the additional barrier height at low and high energies.

Furthermore it turns out that $\Gamma_{0}(e)$ indeed shows an exponential dependence of energy. Interestingly, $\Gamma_{0}\left(e_{\text {cross }}\right) \approx 1 / 20 \mathrm{fs}^{-1}$ is of the order of typical molecular time scales. This also suggests that the increase below $e_{\text {cross }}$ is due to entropic reasons.

The PEL parameters, obtained from the fitting, are listed in Tab!. Note that if not mentioned otherwise from now on all energies are expressed relative to $e_{0, e f f}$, i.e. the maximum of $G_{e f f}(e)$. For the analytical calculations, to be presented below, it is convenient to exclusively use Eq,21, i.e. using $e<e_{\text {cross }}$ and $\tilde{\beta}>0$. The first relation starts to be very well fulfilled if $e_{\text {cross }}-\langle e(T)\rangle>\sigma$ which roughly implies $T<0.6$ in case of BMLJ and $T<3600$ $\mathrm{K}$ in case of $\mathrm{BKS}-\mathrm{SiO}_{2}$. In this temperature range one also has $\tilde{\beta}>0$.

Interestingly, $e_{\text {cross }}$ is significantly smaller than $e_{0, e f f}$. As will become clear below this difference is crucial for properties like the fragility. The additional barrier height $V_{0}$ is present both for BKS-SiO 2 and BMLJ (and has similar height after normalization by $\sigma$ ). Therefore $V_{0}$ cannot be of any relevance for the question of fragility. It can be directly extracted from the high-temperature behavior.

The observation $\lambda<1$ suggests than even these small systems are not elementary. This is equivalent to the result reported in [21] that a consistent mapping on an elementary trap 
model is not possible.

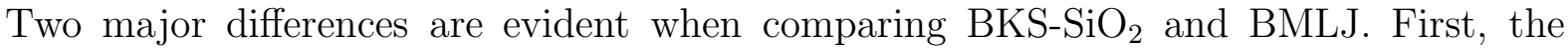
low-energy cutoff for $\mathrm{BKS}-\mathrm{SiO}_{2}$ is significantly larger than the cutoff, dictated by entropy. Thus, the amorphous ground-state is a finite-entropy state. Second, $\left(-e_{\text {cross }}\right) / \sqrt{\lambda \sigma^{2}}$ is much lower for $\mathrm{BKS}-\mathrm{SiO}_{2}$. This means that activated processes become relevant only for states much lower in the PEL. As a consequence, a characteristic temperature like $T_{M C T}$ should be lower for silica than for BMLJ. Indeed, $\Delta\left(\sigma / T_{M C T}\right) \equiv\left(\sigma / T_{M C T}\right)_{\text {silica }}-\left(\sigma / T_{M C T}\right)_{B M L J} \approx$ $12.2-6.7=5.5[54,55]$ and $\left.\Delta\left(-e_{\text {cross }}\right) / \sigma\right)=6.4$ are similar. Furthermore, the energydependence of $\Gamma_{0}(e)$ for $\mathrm{BKS}_{-} \mathrm{SiO}_{2}$ is much more prominent.

\section{THE DYNAMICS OF IDEAL GAUSSIAN GLASS-FORMING SYSTEMS}

\section{A. General}

The MB dynamics can be characterized by a waiting time distribution $\varphi(\tau)$ [20]. From this one can calculate the different moments $\left\langle\tau^{n}\right\rangle$ of $\varphi(\tau)$. It has been shown in previous work that the diffusion constant $D$ is proportional to $1 /\langle\tau\rangle[19]$. Within the continuous-time random walk (CTRW) formalism the structural relaxation time $\tau_{\alpha}$ can be identified with $\left\langle\tau^{2}\right\rangle /\langle\tau\rangle$ [56]. Actually, very recently it has been shown [29, 57] that it is indeed fully justified to use the CTRW-formalism to describe the dynamics of the BMLJ $(N=65)$ system.

Given the distribution of energies as well as the relation between energy and mobility one may ask whether one can explicitly calculate $\left\langle\tau^{n}\right\rangle$. For this purpose we first introduce $\varphi(e)$ as the probability density that in a series of different MB, visited by the system, a randomly chosen MB has energy $e$. Then the average waiting time is given by averaging $\langle\tau(e)\rangle$ over all MB, i.e.

$$
\langle\tau\rangle=\int \operatorname{de} \varphi(e)\langle\tau(e)\rangle=\int \operatorname{de} \varphi(e) / \Gamma(e) .
$$

$\varphi(e)$ is distinctly different from the Boltzmann distribution $p_{e q}(e)$ which denotes that at a randomly given time the present $\mathrm{MB}$ has energy $e$, i.e. $p_{e q}(e) \propto \varphi(e)\langle\tau(e)\rangle$. Including a normalization factor this can be rewritten as

$$
p_{e q}(e)=\frac{\varphi(e)}{\Gamma(e)\langle\tau\rangle}
$$

Qualitatively, this relation expresses that low-energy states (small $\Gamma(e)$ ) are often observed 
(at randomly chosen times) although their actual number $\propto \varphi(e)$ may be very small. Multiplication of Eq25 with $\Gamma(e)$ and subsequent integration yields

$$
\langle\tau\rangle^{-1}=\int \operatorname{de} p_{e q}(e) \Gamma(e) \equiv\langle\Gamma\rangle_{p}
$$

Thus, the average waiting time is also related to the rate average over the equilibrium probability distribution. Note the different notations $\left(\langle\right.$.$\rangle as the \varphi$-average vs. $\langle.\rangle_{p}$ as the $p$-average. Using the explicit form of $G_{e f f}(e)$ one obtains after a straightforward integration

$$
\langle\tau\rangle^{-1}=\Gamma_{0} \exp \left(\left(\lambda^{2} / 2-\lambda\right) \mu^{2} / 2\right) \exp \left(-\beta V_{0}\right)
$$

So far no information about the nature of the relaxation process has entered the analysis. In the most simple case the escape from a state with energy $e$ is governed by a single barrier height. Then the waiting time distribution, related to this energy, is just $\Gamma(e) \exp (-\Gamma(e) t)$. For the BMLJ $(\mathrm{N}=65)$ system one has $1 / \lambda \approx 2$ subsystems. In the most simple picture the total energy is then the sum of two independent subsystems, each with energy $e_{i}\left(e_{1}+e_{2}=e\right)$ and for a given energy decomposition the total rate $\Gamma(e)$ is given by $\Gamma\left(e_{1}\right)+\Gamma\left(e_{2}\right)$. Actually, as outlined in [29], the normalized second moment $\left\langle\tau(e)^{2}\right\rangle /\langle\tau(e)\rangle^{2}$ is expected to be around 16 for $T=0.5$ for 2 subsystems as compared to 2 for an elementary system. The broadening of the waiting time distribution at fixed energy is due to the fact that for a given total energy $e$ several decompositions $e=e_{1}+e_{2}$ are possible, each giving rise to different escape rates. The numerically observed value is approx. 8 [58]. This means that the BMLJ(N=65) system behaves, to first approximation, like two independent subsystems (each described by $\lambda=1$ and variance $\sigma^{2} / 2$ if $\sigma^{2}$ is the variance of the original system). A possible reason for the decrease of 16 to 8 will be given below. In any event, in what follows we neglect this effect and postulate that the elementary system behaves like an IGGF with $\lambda=1$ and an exponential waiting time distribution at given energy. Since the waiting time distribution at fixed energy is a well-defined observable in the MB approach the subsequent calculations could be easily generalized to take into account deviations from a purely exponential behavior of the waiting time distribution of the elementary system.

This aspect is strongly related with the old discussion of homogeneous vs. heterogeneous relaxation [59, 60]. Heterogeneous relaxation would simply mean that one has a superposition of exponentially relaxing entities. Experimentally it has been shown that the dynamics at the glass transition is basically heterogeneous [61]. This indicates that the choice of an exponential waiting time distribution is indeed not too bad. 


\section{B. Calculation of moments}

With this approximation the waiting time distribution $\varphi(\tau)$ and the distribution $\varphi(e)$, reflecting the thermodynamics, are related via

$$
\varphi(\tau) \propto \int d e \int d t \varphi(e) \exp (-\Gamma(e) t) \delta(t-\tau) .
$$

Its different moments $\left\langle\tau^{n}\right\rangle$ can be directly calculated

$$
\left\langle\tau^{n}\right\rangle=\int \operatorname{de} \varphi(e) n ! \Gamma(e)^{-n}=n !\langle\tau\rangle\left\langle\Gamma^{1-n}\right\rangle_{p} \exp \left(n \beta V_{0}\right) .
$$

For the second equality Eq.25 has been employed.

Straightforward evaluation of Gaussian integrals yields

$$
\left\langle\left(\Gamma / \Gamma_{0}\right)^{m}\right\rangle_{p}=\exp \left[\left(m^{2} / 2-m\right) \mu^{2}-m \beta V_{0}\right] .
$$

The case $m=1$ recovers Eq27, Furthermore, the case $m=-1$ gives finally rise to

$$
\left\langle\tau^{2}\right\rangle /\langle\tau\rangle^{2}=\exp \left(\mu^{2}\right)
$$

In most models no distinction between $e_{\text {cross }}$ and $e_{0, e f f}$ is made. Then $\mu$ can be identified with $\beta$. The relations for this special case can be already found in literature [36]. Note that in this limit Eq27 corresponds to the well-known $1 / T^{2}$ temperature dependence, discussed, e.g., in [50].

\section{APPLICATIONS}

\section{A. Kinetic fragility}

Here we analyse the temperature dependence of $\langle\tau\rangle$ (and thus of $D(T)$ ) and in particular the fragility. The glass transition temperature is defined by the condition

$$
\Gamma_{0}\left\langle\tau\left(T_{g, K}\right)\right\rangle=10^{K} .
$$

Neglecting for the time being the somewhat different temperature behavior of $D(T)$ and $\eta(T)$ (see below) $T_{g, 16}=1 / \beta_{g, 16}$ roughly corresponds to the calorimetric $T_{g}$ because $\eta\left(T_{g}\right) / \eta(T \gg$ $\left.T_{g}\right) \approx 10^{16}$. Simple expressions emerge for the case $V_{0}=0$ (corrections can be simply calculated but only mildly influence the results). Using Eq 30 a simple calculation yields

$$
\sigma \beta_{g, K}=k_{\text {entro }} \sigma+\sqrt{2 K \ln (10)} .
$$




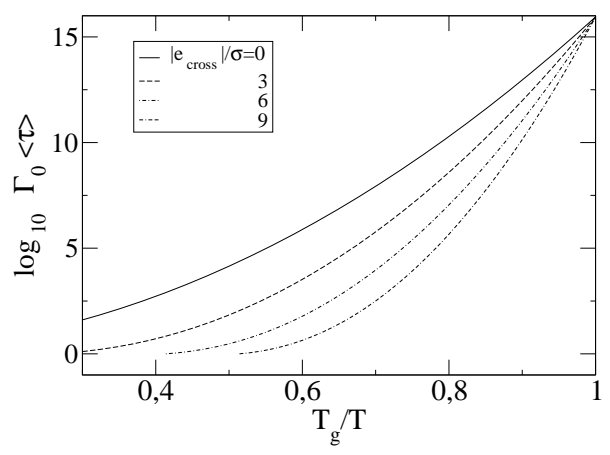

Figure 4: The temperature dependence of $\Gamma_{0}\langle\tau\rangle(\propto D(T))$ for different values of the crossover energy with $\lambda=1$ (the values are given with respect to $e_{0, e f f}$ ).

In relation to the definition of $T_{g}$ we use the notion $m_{k i n, K}$ rather than $m_{k i n}$ (see Eq. 3 ) to express the dependence on the time scale. Then a straightforward calculation yields

$$
m_{k i n, K}=2 K+\sqrt{2 K / \ln (10)} k_{\text {entro }} \sigma .
$$

In this regime the fragility depends on the dimensionless parameter $k_{\text {entro }} \sigma=-e_{\text {cross }} / \sigma$. Thus, the dynamic crossover energy is a central PEL parameter determining the fragility. These results are visualized in Fig, 4 . One can clearly see how the fragility increases with increasing $-e_{\text {cross }} / \sigma$.

Note that Eq 34 implies that BMLJ is stronger than $\mathrm{BKS}_{3} \mathrm{SiO}_{2}$ if the cutoff were artificially removed so that the PEL is purely Gaussian. The non-fragile behavior of BMLJ has been already mentioned in Ref.[62].

Of course, since the temperature dependence of $\tau_{\alpha}$ is in general not identical to that of $\langle\tau\rangle$ the results would slightly differ if $m_{k i n, K}$ is calculated for $\tau_{\alpha}$ or $\eta$ rather than for the diffusivity.

Empirical relations to correlate the fragility with, e.g., the Poisson ratio have been suggested [63] but are questioned in [64]. It would be interesting to check whether there exists a physical connection between the observables, suggested in that work, and the value of $e_{\text {cross }}$, determining the crossover from liquid-like behavior to solid-like behavior. 


\section{B. Relation to the AG approach}

Alternatively, one can calculate the value of $\beta_{g}$ under the assumption of the AG relation Eq.1 and a Gaussian PEL (using $\beta_{\text {harm }}=0$ ). Then one has to solve the equation

$$
10^{K}=\exp \left(\beta_{g} B_{A G} /\left(\alpha-\beta_{g}^{2} \sigma^{2} / 2 N\right)\right.
$$

For large $K$ one obtains

$$
\beta_{g}=\frac{\sqrt{2 \alpha N}}{\sigma}-\frac{B_{A G} \sqrt{N}}{\sigma K \ln (10)} .
$$

Then a straightforward calculation yields for the fragility (again in the limit of large $K$ )

$$
m_{k i n, K}=\frac{\sqrt{2 \alpha} K^{2}(\ln 10)^{2} \sigma}{B_{A G} \sqrt{N}} .
$$

Within the AG-approach the fragility depends on the density of states, i.e. $\alpha$, as well as the empirical constant $B_{A G}$. A large number of states implies larger fragility (at least for fixed $B_{A G}$ which, of course, could also depend on $\alpha[25]$ ).

It may be interesting to compare this relation with the fragility Eq. 34 , obtained for an IGGF. Qualitatively, both relations would show a somewhat similar behavior if systems with large $\alpha$ are related to systems with a low crossover energy $e_{\text {cross }}$, i.e. large $k_{\text {entro }}$. This is not unreasonable because in the spirit of percolation-like arguments for a larger number of IS the system would be able to find a path with a lower barrier to move between two low-energy IS. However, in a strict way it will not be possible to map Eq.37 on Eq.34 because of the different $K$-dependence. Formally, this problem could be solved if $\alpha$ decreases with increasing $K$, i.e. going to longer time scales and thus lower glass transition temperatures. Qualitatively this statement is equivalent to the requirement that $G(e)$ decays faster than a Gaussian. This has been suggested in [65]. Physically this might, e.g., occur as a consequence of a broadened low-energy cutoff.

\section{Thermodynamic fragility}

In the spirit of the thermodynamic fragility as discussed in [23, 24] one can define the thermodynamic fragility index via [25]

$$
m_{\text {thermo }, K}=-\beta_{g} \frac{S_{c}^{\prime}\left(\beta_{g}\right)}{S_{c}\left(\beta_{g}\right)} .
$$


We obtain, using Eq.9,

$$
m_{\text {thermo }, K}=\frac{\sigma^{2}\left(\beta_{g}-\beta_{\text {harm }}\right) \beta_{g}}{N \alpha-\sigma^{2}\left(\beta_{g}-\beta_{\text {harm }}\right)^{2} / 2} .
$$

Note that the denominator must be positive because otherwise the entropy of the system would be negative. Under this condition, an increase of $\sigma k_{\text {entro }}$ (which is the only relevant dimensionless parameter, characterizing IGGF) and thus of $\sigma \beta_{g}$ (via Eq. 33), gives rise to an increase of $m_{\text {thermo }}$ and $m_{k i n}$, independent of the values of $\beta_{g}$ or $\alpha$. This strong correlation of $m_{\text {kin }}$ and $m_{\text {thermo }}$ is in agreement with the experimental observation for most systems [23].

Interestingly, increasing the value of $\beta_{\text {harm }}$ yields a decrease in $m_{\text {thermo,K}}$. However, a different behavior emerges if one includes the vibronic contribution into the entropy, i.e. by using $S_{\text {ex }}(T)=S_{c}(T)+S_{\text {harm }}(T)$ rather $S_{c}(T)$. A straightforward calculations yields $S_{\text {ex }}\left(T, \beta_{\text {harm }}\right)=S_{c}\left(T,-\beta_{\text {harm }}\right)$, thereby neglecting a constant and a term, depending loga-

rithmically on $\beta$ [29]. Accordingly, when defining $m_{k i n, K}$ on the basis of $S_{e x}(T)$ one obtains an increasing thermodynamic fragility for increasing $\beta_{\text {harm }}$ in agreement with the qualitative discussion in [23].

If the cutoff starts to influence the system a detailed calculation is no longer possible because the behavior of the configurational entropy at low temperatures depends on the details of $G(e)$ at low energies. Thus, it is not surprising that for $\mathrm{SiO}_{2}$ the thermodynamic fragility does not follow the general trend [23].

The present discussion complements the work by [41] where the kinetic and the thermodynamic fragility have been discussed with reference to the AG-relation. Simulations have also revealed a significant correlation between both fragilities.

\section{Relaxation properties}

Here we ask for the probability $S_{0}(t)$ that a system in equilibrium has not performed a hopping process until time $t$. It is given by

$$
S_{0}(t)=\int d e p_{e q}(e) \exp (-\Gamma(e) t)
$$

In what follows the trivial factor $\exp \left(\beta V_{0}\right)$ will be omitted. For sufficiently low temperatures the decay of this function can be related to the structural relaxation [56, 57].

As shown in [29, 66] one can approximate for intermediate times $\left(S_{0}(t) \approx 1 / e\right)$

$$
S_{0}(t) \approx \exp \left(-\left(t / \tau_{K W W}\right)^{\beta_{K W W}}\right)
$$


with

$$
\beta_{K W W}=1 / \sqrt{1+\mu^{2}}
$$

and $\tau_{K W W}=1 / \Gamma^{\star}$ where

$$
\Gamma^{\star}=\Gamma(\langle e(T)\rangle)=\exp \left(-\mu^{2}\right)
$$

This may justify the use of the stretched exponential as a fitting function at least for intermediate times. This result is insensitive to the specific form of $\Gamma(e)$ since $\Gamma(e)$ only enters via $\Gamma^{\star}$. Note that for the IGGF the non-exponentiality tends to increase when going to lower temperatures. Furthermore one can show that in very long-time decay is algebraic [29, 66]

$$
S_{0}(t) \propto t^{-u / 2 \mu^{2}}
$$

One can define the $\alpha$-relaxation time $\tau_{\alpha}$ via

$$
\tau_{\alpha}=\int d t S_{0}(t)
$$

which corresponds to the typical time until a particle jumps for the first time [56, 57]. From Eq. 40 one immediately obtains (also using Eq.

$$
\tau_{\alpha}=\left\langle\Gamma^{-1}\right\rangle_{p}=\left(1 / \Gamma_{0}\right) \exp \left(3 \mu^{2} / 2\right)
$$

This has to be compared with the average hopping time $\langle\tau\rangle$ (Eq.27). One obtains

$$
\tau_{\alpha} /\langle\tau\rangle=\exp \left(\mu^{2}\right)
$$

Since the left side is proportional to $D \tau_{\alpha}$ Eq,47 expresses the invalidation of the StokesEinstein relation for IGGF. Using the definition of the exponent $a$ via $D(T) \tau_{\alpha}(T) \propto \tau_{\alpha}^{a}$, i.e. $\langle\Gamma\rangle\left\langle\Gamma^{-1}\right\rangle \propto\left\langle\Gamma^{-1}\right\rangle^{\alpha}$ one obtains $a=2 / 3$. Experimental values are smaller (e.g. 0.25 for orthoterphenyl [27] and 0.23 for TNB [67]). Thus, the decoupling seems to be too strong. Qualitatively the strong increase of $\tau_{\alpha}$ with decreasing temperature is due to the very longtime tail of $S_{0}(t)$.

\section{DISCUSSION AND SUMMARY}

The IGGF has been introduced, based on the numerical results for BMLJ and BKS$\mathrm{SiO}_{2}$ (except for the low-energy cutoff for $\mathrm{BKS}-\mathrm{SiO}_{2}$ ) at small system sizes. The general 
concepts are also compatible with several models proposed to rationalize the dynamics of supercooled liquids. Thus, one naturally finds how properties such as the non-exponentiality are generated.

More specifically, the key conclusions are as follows: 1.) If the cutoff-energy does not interfere the temperature-dependence of the dynamics is fully captured by the value of $\mu$ (except for a trivial $\exp \left(-\beta V_{0}\right)$-term). This means in particular that at $T_{g}$ an IGGF has a fixed value of $\mu$, independent of $\sigma k_{\text {entro }}$ and thus independent of its fragility. This implies via Eq.42 that the stretching parameter $\beta_{K W W}$ does not depend on the fragility if determined exactly at $T_{g}$. This may rationalize the weak correlation between $\beta_{K W W}$ and $m_{k i n}$ for the molecular glass-forming systems, as mentioned above. Of course, residual fluctuations are expected when the smaller-order effects of $\lambda, \kappa$ and $V_{0}$ are taken into account. 2.) The fragility of a system is to a large extent dominated by the crossover energy $e_{\text {cross }}$ relative to the width of the energy distribution, i.e. $\sigma$. Systems are more fragile if the crossover from solid-like activated dynamics to liquid-like non-activated dynamics occurs at low energies, relative to the width of $G(e)$. Of course, as soon as the low-energy cutoff of the PEL comes into play (such as for BKS-SiO${ }_{2}$ ) the system automatically behaves Arrhenius-like and thus is classified as strong. This also shows that the fragility is only partly able to classify a glassforming system because already the present discussion shows that there at least two very different parameters, $e_{\text {cut }}$ and $e_{\text {cross }}$, which strongly influence the fragility. 3.)Although the BMLJ data can be fit to the AG-relation, from a conceptual point of view the IGGF is not compatible with the AG-relaxation. This can be seen from the different dependence of the fragility on $K$. On a qualitative level this discrepancy could be reduced if the distribution of states decays stronger than a Gaussian at the low-energy end. 4.) The thermodynamic fragility indeed is correlated with the kinetic fragility, albeit in a non-trivial way. Again, the systems with a cutoff-behavior (most notably $\mathrm{BKS}-\mathrm{SiO}_{2}$ ) have to be discussed separately. 5.) Finally, the IGGF displays non-exponential relaxation with a non-exponentiality which increases with decreasing temperature and, in agreement with the experiment, shows a violation of the Stokes-Einstein relation.

Conceptually, the presence of individual relaxation processes naturally is attributed to small systems, reflecting the typical length scales of cooperative dynamics during single MB transitions. Thus, any strict comparison with simulations in the framework of the PEL approach is conveniently performed with small systems. As shown in previous work the 
diffusion constant as well as the thermodynamic properties of the BMLJ $(N=65)$-system only have very minor finite-size effects when comparing with the results obtained for much larger systems [31, 68, 69]. However, the structural relaxation time as the well as the nonexponentiality has somewhat larger finite-size effects [69]. This effect can be understood if one assumes a specific type of coupling between adjacent subsystems of a larger system. When some subsystem relaxes it may change the mobility of the adjacent subsystems [29]. A similar idea can be already found in [36, 70] and has been also implemented in the context of the rate memory to explain the results of multidimensional NMR experiments [48, 71, 72, 73]. In this way the very immobile regions typically become mobile at some stage and can relax subsequently. In some sense this idea is also related to the philosophy of the facilitated spin models where the local mobility is also influenced by the state of the neighbor spins [74, 75, 76]. The coupling between adjacent subsystems can be formulated such that the diffusion constant and the thermodynamics does not change whereas the structural relaxation time, all moments $\left\langle\tau^{n}\right\rangle$ for $n \geq 2$ and the degree of non-exponentiality decrease upon this coupling [29]. This might also explain why the second moment for the BMLJ system is by a factor of 2 smaller than expected (see above). In particular the exponent $a$, characterizing the violation of the Stokes-Einstein equation approaches experimentally relevant values [29]. However, one of the key results, namely the utmost relevance of a single dimensionless parameter $\mu$ would still be valid. In any event, the path back from small systems to macroscopic systems is one of the challenges for future work. Using the IGGS as the elementary system for such models is definitely a reasonable starting point.

We gratefully acknowledge important input from C. Rehwald and O. Rubner as well as very helpful correspondence with L. Berthier about this topic.

[1] P. G. Debenedetti and F. H. Stillinger, Nature 410, 259 (2001).

[2] K. Binder and W. Kob, Glassy materials and disordered solids (World Scientific, 2005).

[3] J. C. Dyre, Rev. Mod. Phys. 78, 953 (2006).

[4] V. Lubchenko and P. G. Wolyness, Ann. Rev. Phys. Chem. 58, 235 (2006).

[5] M. Hurley and P. Harrowell, Phys. Rev. E 52, 1694 (1995).

[6] W. Kob, C. Donati, S. J. Plimpton, P. H. Poole, and S. C. Glotzer, Phys. Rev. Lett. 79, 2827 
(1997).

[7] C. Donati, J. F. Douglas, W. Kob, S. J. Plimpton, P. H. Poole, and S. C. Glotzer, Phys. Rev. Lett. 80, 2338 (1998).

[8] C. Donati, S. C. Glotzer, P. H. Poole, W. Kob, and S. J. Plimpton, Phys. Rev. E 60, 3107 (1999).

[9] C. Donati, S. C. Glotzer, and P. H. Poole, Phys. Rev. Lett. 82, 5064 (1999).

[10] A. Heuer and K. Okun, J. Chem. Phys. 106, 6176 (1997).

[11] J. Qian, R. Hentschke, and A. Heuer, J. Chem. Phys. 110, 4514 (1999).

[12] D. J. Wales, Energy landscapes (Cambridge University Press, 2003).

[13] F. Sciortino, J. Stat. Mech. P05015 (2005).

[14] F. H. Stillinger and T. A. Weber, Phys. Rev. A 25, 978 (1982).

[15] F. H. Stillinger and T. A. Weber, Science 225, 983 (1984).

[16] T. B. Schrøder, S. Sastry, J. C. Dyre, and S. C. Glotzer, J. Chem. Phys. 112, 9834 (2000).

[17] F. H. Stillinger, Science 267, 1935 (1995).

[18] T. F. Middleton and D. J. Wales, Phys. Rev. B 64, 024205 (2001).

[19] B. Doliwa and A. Heuer, Phys. Rev. E 67, 030501 (2003).

[20] B. Doliwa and A. Heuer, Phys. Rev. E 67, 031506 (2003).

[21] R. A. Denny, D. R. Reichman, and J. P. Bouchaud, Phys. Rev. Lett. 90, 025503 (2003).

[22] G. Adam and J. H. Gibbs, J. Chem. Phys. 43, 139 (1965).

[23] L. Martinez and C. Angell, Nature 410, 663 (2001).

[24] L.-M. Wang, C. A. Angell, and R. Richert, J. Chem. Phys. 125, 074505 (2006).

[25] G. Ruocco, F. Sciortino, F. Zamponi, C. De Micheleand T. Scopigno, J. Chem. Phys. 120, 10666 (2004).

[26] C. A. Angell, J. Phys.-Condes. Matter 12, 6463 (2000).

[27] F. Fujara, B. Geil, H. Sillescu, and G. Fleischer, Z. Phys. B 88, 195 (1992).

[28] R. Bohmer, K. L. Ngai, C. A. Angell, and D. J. Plazek, J. Chem. Phys. 99, 4201 (1993).

[29] A. Heuer, (submitted) (2008).

[30] F. Sciortino, W. Kob, and P. Tartaglia, Phys. Rev. Lett. 83, 3214 (1999).

[31] S. Buechner and A. Heuer, Phys. Rev. E 60, 6507 (1999).

[32] F. W. Starr, S. Sastry, E. La Nave, A. Scala, H. E. Stanley, and F. Sciortino, Phys. Rev. E 63, 041201 (2001). 
[33] E. L. Nave, S. Mossa, F. S. F, and P. Tartaglia, J. Chem. Phys. 120, 6128 (2000).

[34] A. Saksaengwijit, J. Reinisch, and A. Heuer, Phys. Rev. Lett. 93, 235701 (2004).

[35] B. Doliwa and A. Heuer, Phys. Rev. Lett. 91, 235501 (2003).

[36] C. Monthus and J. P. Bouchaud, J. Phys. A-Math. Gen. 29, 3847 (1996).

[37] F. Sciortino, W. Kob, and P. Tartaglia, Phys. Rev. Lett. 83, 3214 (1999).

[38] S. Buechner and A. Heuer, Phys. Rev. Lett. 84, 2168 (2000).

[39] A. Saksaengwijit and A. Heuer, Phys. Rev. E 74, 051502 (2006).

[40] I. Saika-Voivod, F. Sciortino, and P. H. Poole, Phys. Rev. E. 69, 041503 (2004).

[41] S. Sastry, Nature 409, 164 (2001).

[42] S. Mossa, E. La Nave, H. E. Stanley, C. Donati, F. Sciortino, and P. Tartaglia, Phys. Rev. E 65, 041205 (2002).

[43] N. Giovambattista, H. E. Stanley, and F. Sciortino, Phys. Rev. Lett. 91, 115504 (2003).

[44] F. Sciortino, E. L. Nave, and P. Tartaglia, Phys Rev. Lett. 91, 155701 (2003).

[45] S. Brawer, J. Chem. Phys. 81, 954 (1984).

[46] J. C. Dyre, Phys. Rev. Lett. 58, 792 (1987).

[47] V. Arkhipov and H. Baessler, J. Phys. Chem. 98, 662 (1994).

[48] G. Diezemann, J. Chem. Phys. 107, 10112 (1997).

[49] G. Diezemann, H. Sillescu, G. Hinze, and R. Bohmer, Phys. Rev. E 57, 4398 (1998).

[50] J. C. Dyre, Phys. Rev. B 51, 12276 (1995).

[51] S. Brawer, Relaxation in Viscous Liquids and Glasses (The American Ceramic Society, Inc., $1985)$.

[52] A. Saksaengwijit and A. Heuer, Phys. Rev. E 73, 061503 (2006).

[53] V. de Souza and D. Wales, Phys. Rev. Lett. 96, 057802 (2006).

[54] E. La Nave, H. E. Stanley, and F. Sciortino, Phys. Rev. Lett 88, 035501 (2002).

[55] B. Doliwa and A. Heuer, Phys. Rev. E. 67, 031506 (2003).

[56] L. Berthier, D. Chandler, and J. Garrahan, Europhys. Lett. 69, 320 (2005).

[57] O. Rubner and A. Heuer, (in preparation) (2007).

[58] A. Heuer, B. Doliwa, and A. Saksaengwijit, Phys. Rev. E 72, 021503 (2005).

[59] K. Schmidt-Rohr and H. Spiess, Phys. Rev. Lett. 66, 3020 (1991).

[60] R. Richert, Chem. Phys. Lett. 216, 223 (1993).

[61] R. Bohmer, R. V. Chamberlin, G. Diezemann, B. Geil, A. Heuer, G. Hinze, S. C. Kuebler, 
R. Richert, B. Schiener, H. Sillescu, et al., J. Non-Cryst. Solids 235, 1 (1998).

[62] G. Tarjus, D. Kivelson, and P. Viot, J. Phys.: Cond. Mat. 12, 6497 (2000).

[63] V. H. Novikov and A. P. Sokolov, Nature 431, 961 (2003).

[64] S. N. Yannopoulos and G. P. Johari, Nature 442, E7 (2006).

[65] D. Matyushov and C. Angell, J. Chem. Phys. 123, 034506 (2005).

[66] B. Castaing and J. Souletie, J. Phys. I 1, 403 (1991).

[67] S. Swallen, P. Bonvallet, R. McMahon, and M. Ediger, Phys. Rev. Lett. 90, 015901 (2003).

[68] B. Doliwa and A. Heuer, J. Phys. C: Cond. Mat. 15, S849 (2003).

[69] D. A. Stariolo and G. Fabricius, J. Chem. Phys. 125, 064505 (2006).

[70] J. P. Bouchaud, A. Comtet, and C. Monthus, J. Phys. I 5, 1521 (1995).

[71] A. Heuer, M. Wilhelm, H. Zimmermann, and H. Spiess, Phys. Rev. Lett. 75, 2851 (1995).

[72] H. Sillescu, J. Chem. Phys. 104, 4877 (1996).

[73] A. Heuer, Phys. Rev. E 56, 730 (1997).

[74] G. Fredrickson and H. Andersen, Phys. Rev. Lett. 53, 1244 (1984).

[75] J. P. Garrahan and D. Chandler, Phys. Rev. Lett. 89, 035704 (2002).

[76] L. Berthier and J. P. Garrahan, Phys. Rev. E. 68, 041201 (2003). 